\title{
Preoperative Mapping of the Supplementary Motor Area in Patients with Brain Tumor Using Resting-State fMRI with Seed-Based Analysis
}

\author{
(D). Wongsripuemtet, (D)A.E. Tyan, (D) A. Carass, DS. Agarwal, DS.K. Gujar, (D).J. Pillai, and (D).I. Sair
}

\begin{abstract}
BACKGROUND AND PURPOSE: The supplementary motor area can be a critical region in the preoperative planning of patients undergoing brain tumor resection because it plays a role in both language and motor function. While primary motor regions have been successfully identified using resting-state fMRI, there is variability in the literature regarding the identification of the supplementary motor area for preoperative planning. The purpose of our study was to compare resting-state fMRI to task-based fMRI for localization of the supplementary motor area in a large cohort of patients with brain tumors presenting for preoperative brain mapping.
\end{abstract}

MATERIALS AND METHODS: Sixty-six patients with brain tumors were evaluated with resting-state fMRI using seed-based analysis of hand and orofacial motor regions. Rates of supplementary motor area localization were compared with those in healthy controls and with localization results by task-based fMRI.

RESULTS: Localization of the supplementary motor area using hand motor seed regions was more effective than seeding using orofacial motor regions for both patients with brain tumor $(95.5 \%$ versus $34.8 \%, P<.001)$ and controls $(95.2 \%$ versus $45.2 \%, P<.001)$. Bilateral hand motor seeding was superior to unilateral hand motor seeding in patients with brain tumor for either side $(95.5 \%$ versus $75.8 \% / 75.8 \%$ for right/left, $P<.001)$. No difference was found in the ability to identify the supplementary motor area between patients with brain tumors and controls.

CONCLUSIONS: In addition to task-based fMRI, seed-based analysis of resting-state fMRI represents an equally effective method for supplementary motor area localization in patients with brain tumors, with the best results obtained with bilateral hand motor region seeding.

ABBREVIATIONS: $\mathrm{DCS}=$ direct cortical stimulation; rs-fMRI = resting-state fMRI; SMA = supplementary motor area; tb-fMRI = task-based fMRI

$\mathbf{P}$ recise brain function localization is critical for neurosurgical procedures in which lesions are near the eloquent cortex. While direct cortical stimulation (DCS) is the criterion standard for assessing brain function, preoperative noninvasive functional brain imaging can aid in operative planning and decrease operative time. ${ }^{1-4}$ Task-based fMRI ( $\left.t b-f M R I\right)$, a noninvasive technique for examining brain function, demonstrates good correlation with DCS for identification of eloquent brain regions, particularly in areas of sensorimotor and language representation. ${ }^{5,6}$

Received January 26, 2018; accepted after revision May 8.

From the Russell H. Morgan Department of Radiology and Radiological Sciences (J.W., A.E.T., S.A., S.K.G., J.J.P., H.I.S.) and Department of Neurosurgery (J.J.P.), Johns Hopkins University School of Medicine, Baltimore, Maryland; Department of Radiology (J.W.), Siriraj Hospital, Mahidol University, Bangkok, Thailand; and Department of Computer Science and Department of Electrical and Computer Engineering (A.C.), Johns Hopkins University, Baltimore, Maryland.

Please address correspondence to Haris Sair, MD, Russell H. Morgan Department of Radiology and Radiological Science, 600 N Wolfe St, Phipps B-100, Baltimore, MD 21287; hsair1@jhmi.edu; @hsairmd

http://dx.doi.org/10.3174/ajnr.A5709
Thus, fMRI is a valuable clinical tool for preoperative brain function localization.

There are, however, limitations to tb-fMRI, including dedicated hardware and software, trained personnel to administer tasks, designing a suitable task to interrogate a specific brain function, and, most important, patient cooperation, which may be difficult for subsets of patients with cognitive impairment or advanced neurologic disease. Resting-state fMRI (rs-fMRI) can address some of these limitations. ${ }^{7}$ Instead of limiting interrogation to a specific brain function interrogated by a particular task, multiple brain networks can be assessed with a single rs-fMRI acquisition. Because no specific task needs to be performed, rs-fMRI can be more readily applied in patients with neurologic deficits or cognitive disturbance and in young children. Finally, intrinsic brain networks have been shown to be present even during sleep or anesthesia, opening the possibility of using this technique in patients who otherwise may not be able to tolerate MR imaging. ${ }^{8,9}$

The sensorimotor network has been well-studied using rsfMRI in the preoperative setting, ${ }^{10}$ with good agreement of 
rs-fMRI with both tb-fMRI ${ }^{11}$ and DCS. ${ }^{12}$ While the sensorimotor network proper is readily characterized in these studies, there is, however, variability in depiction of the supplementary motor area (SMA) in these studies; in the study of Kokkonen et al, ${ }^{11}$ the SMA was not reliably demonstrated in all subjects. In clinical tb-fMRI, often there are specific requests to localize the SMA due to adjacent lesions.

Many postoperative brain tumor studies have shown that the degree of neurologic deficit after tumor resection correlates with the extent of resection of SMA regions. ${ }^{9,13}$ Findings of transient contralateral weakness and transient speech deficits, including mutism (in the case of SMA resection in the dominant hemisphere) after resection or manipulation are well-established in the neurosurgical literature. ${ }^{14}$

To date, there are limited studies specifically evaluating the feasibility of rs-fMRI for SMA localization in patients with brain tumors. Vassal et $\mathrm{al}^{15}$ recently published a study characterizing the pre- and postoperative SMA in 6 patients with brain tumors. With small numbers of subjects however, the effects of intersubject variability are limited; indeed, while early reports of rs-fMRI for preoperative language localization showed promise in a small number of subjects, ${ }^{16} 2$ subsequent independent analyses with larger sample sizes demonstrated a wide range of accuracy across subjects. ${ }^{3,17}$ We therefore aimed to evaluate the utility of rs-fMRI using seed-based analysis in localizing the SMA in a larger cohort of patients with brain tumors. We hypothesized that the SMA can be readily identified using rs-fMRI in patients with brain tumors and is comparable with tb-fMRI.

\section{MATERIALS AND METHODS \\ Subjects}

In this institutional review board-approved retrospective study, 113 patients with brain lesions who underwent preoperative functional MR imaging between May 2012 and June 2016 were identified using the Johns Hopkins Hospital radiology information system. Forty-one patients were excluded due to lack of hand motor tb-fMRI and rs-fMRI performed during the same scanning session. Two patients were excluded due to artifacts. Four patients were excluded due to diagnoses other than brain tumors. After exclusion, data from 66 patients were used for analysis.

Additionally, 21 healthy subjects from the Kirby 21 MultiModal Reproducibility Study dataset (F.M. Kirby Center, Kennedy Krieger Institute, Baltimore, Maryland) were included in the study for comparison. ${ }^{18}$

\section{Handedness}

A laterality index for patients was calculated using the Edinburgh Handedness Inventory questionnaire. ${ }^{19}$

\section{Lesion Characterization}

Tumor location for each patient was determined from structural MR imaging. The World Health Organization histologic grade was recorded. Lesion volume (in cubic millimeters) was calculated by manual ROI drawing on T2 fluid-attenuated inversion recovery images using the Medical Image Processing, Analysis and Visualization application. ${ }^{20}$

\section{Image Acquisition}

A 3T Trio Tim system (Siemens, Erlanger, Germany) was used with a 12-channel head coil. Structural images included a 3D T1 sequence $(\mathrm{TR}=2300 \mathrm{~ms}, \mathrm{TI}=900 \mathrm{~ms}, \mathrm{TE}=3.5 \mathrm{~ms}$, flip angle $=$ $9^{\circ}, \mathrm{FOV}=24 \mathrm{~cm}$, acquisition matrix $=256 \times 256 \times 176$, slice thickness $=1 \mathrm{~mm})$ and a $2 \mathrm{D}$ T2 FLAIR sequence $(\mathrm{TR}=9310 \mathrm{~ms}$, $\mathrm{TI}=2500 \mathrm{~ms}, \mathrm{TE}=116 \mathrm{~ms}$, flip angle $=141^{\circ}, \mathrm{FOV}=24 \mathrm{~cm}$, acquisition matrix $=320 \times 240 \times 50$, slice thickness $=3 \mathrm{~mm}$ ).

$2 \mathrm{D}$ gradient-echo-planar imaging was used for blood oxygen level-dependent functional imaging $(\mathrm{TR}=2000 \mathrm{~ms}, \mathrm{TE}=30 \mathrm{~ms}$, flip angle $=90^{\circ}, \mathrm{FOV}=24 \mathrm{~cm}$, acquisition matrix $=64 \times 64 \times$ 33 , slice thickness $=4 \mathrm{~mm}$, slice gap $=1 \mathrm{~mm}$, interleaved acquisition).

Given brain shifts due to mass lesions, tb-fMRI was performed in the brain tumor patient group to improve localization of the SMA. All patients underwent bilateral sequential finger-tapping tasks, and 50/66 patients additionally underwent a tongue motor task involving repetitive vertical tongue movements because their lesions were more in proximity to the ventral motor region. A block design of a 30-second alternation of task and rest was used for both tasks with 90 volumes acquired.

One hundred eighty volumes were acquired for rs-fMRI $(\sim 6$ minutes). All patients were instructed to not move, keep their eyes closed, and not to think of anything during the acquisition.

Imaging details for the Kennedy Krieger Institute dataset are previously described. ${ }^{18}$

\section{Image Analysis and Processing}

FMRI was analyzed using Statistical Parametric Mapping (SPM; (http://www.fil.ion.ucl.ac.uk/spm/software/spm12) as well as custom Matlab (MathWorks, Natick, Massachusetts) scripts.

Both tb-fMRI and rs-fMRI underwent slice-timing correction, motion-correction, coregistration to the T1-weighted images, normalization to the Montreal Neurological Institute-152 template, and spatial smoothing using a 6-mm full width at half maximum Gaussian kernel. For rs-fMRI, additional processing included tagging of outlier volumes based on large shifts in global average signal using the ArtRepair toolbox (http://cibsr.stanford. edu/tools/human-brain-project/artrepair-software.html) in SPM ${ }^{21}$ after motion correction, linear detrending, physiologic nuisance regression using CompCor, ${ }^{22}$ bandpass filtering $(0.01-0.1 \mathrm{~Hz})$ after normalization, and finally removal of outlier volumes tagged by ArtRepair ("scrubbing”).

Standard general linear model analysis was used for tb-fMRI with the canonical hemodynamic response function convolved with a boxcar function. No derivatives, global intensity normalization, or confound matrix was used. A 128-second high-pass filter was used. An autoregressive model was used to account for temporal autocorrelations. A design matrix was constructed to detect activation during the active task state compared with rest. SPM T-contrast maps were generated without clustering or multiple comparison correction as is customary for single-subject clinical data at our institution.

Rs-fMRI seed to whole brain voxel analysis was performed to generate the motor network. We placed 10 -mm cubic ROIs consistently across subjects in the hand motor and orofacial motor regions of the brain as described below. After extraction of the 
mean time-varying signal across time within the ROI, this time course was used to generate Pearson correlation coefficients with time courses of every other voxel, generating a $3 \mathrm{D}$ correlation coefficient map across the entire brain:

1) Hand motor functional areas in bilateral brain hemispheres of each subject were determined by identifying the middle of the hand knob ( $\Omega$-shaped) of the primary motor cortex within the precentral gyri.

2) Bilateral orofacial motor functional areas were identified in the inferolateral part of the precentral gyrus, superior to the Sylvian fissures, and below the imaginary line from the intersection of the inferior frontal sulcus and precentral sulcus on the sagittal view.

The anatomic coordinates were placed by a neuroradiologist with 3 years of experience following subspecialty training and supervised by a neuroradiologist with 6 years of experience, including in functional brain imaging.

\section{SMA Positive-Connectivity Evaluation}

For each patient, 6 whole-brain correlation maps were generated with ROIs in the following areas: 1 ) the left hand motor region, 2) the right hand motor region, 3 ) the left orofacial region, 4) the right orofacial region, 5) bilateral hand motor regions, and 6) bilateral orofacial regions. Final correlation maps were registered to the anatomic image to identify areas of functional connectivity with the SMA region. The SMA was defined as the area in the medial portion of the superior frontal gyrus in front of the primary motor cortex and superior to the cingulate sulcus. Its anterior boundary was defined by a line passing perpendicularly through the rostrum of the corpus callosum. ${ }^{23}$ Identification of the SMA was evaluated by 2 neuroradiologists with specific expertise in fMRI with consensus. Tb-fMRI was used to localize the SMA for confirmation in the patient group.

\section{Statistical Analysis}

The number of cases (as well as the percentage of total cases) in which the SMA was identified from rs-fMRI was recorded and reported for each of the 6 ROI schemes. Considering the dependent design, we used the McNemar $\chi^{2}$ test to assess differences in positive SMA identification when the ROI went from single to bilateral regions. All analysis was performed in $\mathrm{R}$ statistical and computing software (Version 3.2.4; http://www. r-project.org).

\section{RESULTS}

\section{Subjects}

Of the 66 patients, 38 were men and 28 were women, ranging from 18 to 75 years of age (mean, $40.8 \pm 14.6$ years). For the 21 healthy control subjects, there were 11 men and 10 women, from 25 to 61 years of age (mean, $31.8 \pm 9.2$ years).

\section{Handedness}

By means of the laterality index cutoff points of \pm 0.2 (less than $-0.2=$ left-handedness, $>0.2=$ right-handedness, and $-0.2-$ $0.2=$ ambidexterity), in the patient group, the laterality index ranged from -0.72 to 1 (mean $=0.696)$. Sixty-three patients demonstrated right-handedness, and 3 patients demonstrated left-handedness.

For control patients, on the basis of the Kirby 21 Multi-Modal Reproducibility Study data base, 19 demonstrated right-handedness and 2 subjects demonstrated left-handedness. No laterality index information was available from this dataset.

\section{Lesions}

Of 66 patients, 46 had tumors in the left hemisphere ( 24 involving the left frontal lobe); 18 , in the right hemisphere (12 involving the right frontal lobe); 1 , in both frontal lobes; and 1 had multiple lesions throughout both hemispheres. In 7 patients, there was direct tumor involvement of the expected region of the SMA ( 4 on the left and 3 on the right side); in 3 patients, there was involvement of the primary motor cortex.

Fifty-three percent (35/66) of tumors were low-grade gliomas (6 diffuse astrocytomas, 1 fibrillary astrocytoma, 26 oligodendrogliomas, and 2 that had not been histologically confirmed but appeared to be low-grade gliomas on imaging). Forty-two percent (28/66) of the lesions were high-grade gliomas (9 infiltrative astrocytomas, 8 anaplastic astrocytomas, 3 anaplastic oligoastrocytomas, and 8 glioblastomas). The remaining patients were diagnosed with myeloid sarcoma, metastatic melanoma, or metastatic adenocarcinoma. The tumor sizes ranged from $8.4 \times 10^{2}$ to $1.6 \times$ $10^{5} \mathrm{~mm}^{3}\left(\right.$ mean $\left.=4.3 \times 10^{4} \pm 3.7 \times 10^{4} \mathrm{~mm}^{3}\right)$.

The demographics of subjects, location, histopathology, and World Health Organization grading of the lesions are listed in Table 1 .

\section{SMA Identification}

In both the control and patient groups, the SMA was identified more frequently using seeding location from hand motor areas than from orofacial areas, with use of bilateral ROIs superior to the unilateral right or left motor ROI (Figure and Table 2).

In the patients with brain tumors, the SMA was identified in 50/66 (75.8\%) using the left hand motor ROI, 50/66 (75.8\%) using the right hand motor ROI, 63/66 (95.5\%) using the bilateral hand motor ROIs, 18/66 (27.3\%) using the left orofacial ROI, 17/66 (25.8\%) using the right orofacial ROI, and 23/66 (34.8\%) using the bilateral orofacial ROIs (Table 3). A significant difference was seen between using bilateral hand motor ROIs versus either unilateral hand motor ROI $(P<.001)$. In addition, a significant difference was seen between using bilateral hand motor ROIs versus bilateral orofacial ROIs $(P<.001)$ (Table 3).

In the control group, the SMA was identified in 34/42 (81.0\%) scans using the left hand motor ROI, 38/42 (90.5\%) scans using the right hand motor ROI, 40/42 (95.2\%) scans using the bilateral hand motor ROIs, 9/42 (21.4\%) scans using the left orofacial ROI, $14 / 42(33 \%)$ scans using the right orofacial ROI, and $19 / 42$ (45.2\%) scans using the bilateral orofacial ROIs. While no significant difference was found between unilateral and bilateral ROIs for either hand or orofacial motor ROIs after multiple-comparison correction, there was a significant difference between bilateral hand motor ROIs versus bilateral orofacial ROIs (Table 4).

There was no statistically significant difference in the percentage of positive SMA identification between the group of patients with brain tumor and healthy control subjects (Table 5).

AJNR Am J Neuroradiol 39:1493-98 Aug 2018 www.ajnr.org 
Table 1: Demographic and clinical characteristics of patients and controls

\begin{tabular}{|c|c|c|}
\hline Characteristic & $\begin{array}{l}\text { Patients with Brain } \\
\text { Tumor }(n=66)\end{array}$ & $\begin{array}{l}\text { Control Subjects } \\
\quad(n=21)\end{array}$ \\
\hline Age (range) (mean) (yr) & $18-75(40.8 \pm 14.6)$ & $25-61(31.8 \pm 9.24)$ \\
\hline \multicolumn{3}{|l|}{ Sex (No.) } \\
\hline Male & 38 & 11 \\
\hline Female & 28 & 10 \\
\hline Tumor size (mean) $\left(\mathrm{mm}^{3}\right)$ & $\begin{array}{c}8.4 \times 10^{2} \text { to } 1.6 \times 10^{5} \\
\left(4.3 \times 10^{4} \pm 3.7 \times 10^{4}\right)\end{array}$ & - \\
\hline Pathology (No.) & & - \\
\hline Diffuse astrocytoma & 6 & \\
\hline Fibrillary astrocytoma & 1 & \\
\hline Infiltrative astrocytoma & 9 & \\
\hline Anaplastic astrocytoma & 8 & \\
\hline Glioblastoma & 8 & \\
\hline Oligodendroglioma & 26 & \\
\hline Anaplastic oligoastrocytoma & 3 & \\
\hline Myeloid sarcoma & 1 & \\
\hline Metastatic melanoma & 1 & \\
\hline Metastatic adenocarcinoma & 1 & \\
\hline $\begin{array}{l}\text { No pathologic report (low-grade } \\
\text { tumor by imaging) }\end{array}$ & 2 & \\
\hline \multicolumn{3}{|l|}{ WHO classification (No.) } \\
\hline Grade I & 0 & \\
\hline Grade II & 33 & \\
\hline Grade III & 20 & \\
\hline Grade IV & 8 & \\
\hline \multicolumn{3}{|l|}{ Tumor location (No.) } \\
\hline Right hemisphere & 18 & \\
\hline Frontal & 12 (3 SMA, 1 PMC) & \\
\hline Parietal & 1 & \\
\hline Temporal & 1 & \\
\hline Insular & 2 & \\
\hline Frontoparietal & 1 & \\
\hline Frontotemporal & 1 & \\
\hline Left hemisphere & 46 & \\
\hline Frontal & 24 (4 SMA, 2 PMC) & \\
\hline Parietal & 3 & \\
\hline Temporal & 11 & \\
\hline Insular & 4 & \\
\hline Frontoparietal & 2 & \\
\hline Frontotemporal & 1 & \\
\hline Parietotemporal & 1 & \\
\hline Bilateral hemisphere & 1 & \\
\hline Multiple lesions & 1 & \\
\hline
\end{tabular}

Note:-PMC indicates primary motor cortex.

\section{DISCUSSION}

There are operative challenges for brain tumors near the SMA, a functional brain region that is not uniformly anatomically bound according to well-established landmarks in contrast to the primary motor cortex. Resection of the SMA, an area primarily involved in voluntary motor movement, ${ }^{24}$ can result in motor and language deficits. ${ }^{25}$ The degree of neurologic deficit corresponds to the extent of resection: Aphasia without motor impairment was seen with anterior SMA resection, likely extending into languagerelated regions termed "pre-SMA"; and manipulation extending to the posterior SMA was correlated with contralateral paresis, with a small percentage of cases having permanent deficits. ${ }^{13}$ Because of these potential deficits, tb-fMRI has been used to help localize the SMA for operative planning, supplemented with DCS if necessary, though the SMA is often difficult to localize even with DCS.

A recent study using seed-based rs-fMRI was able to demonstrate the SMA in patients with gliomas using seeds placed in the hand motor region. ${ }^{26}$ We extended the investigation in a larger cohort of subjects by comparing the accuracy of SMA detection in patients with brain lesions with controls and used different sites of ROI placement, because the hand motor region may not always be feasible for ROI placement depending on the volume and distribution of brain lesions. We demonstrate that the SMA can be identified in both patients with brain tumor and healthy controls using rs-fMRI with seed-based analysis. In a recent study comparing tb-fMRI primary hand motor activation with rs-fMRI using the task-based activation as the seed region for seed-based analysis, the authors reported that the SMA could be identified from the rs-fMRI but not from the tbfMRI. ${ }^{27}$ In our study, the seed location was blinded to tb-fMRI, thus representing a possible scenario in which rs-fMRI may be used in isolation without tbfMRI activation maps.

Between the patients with brain tumor and healthy controls, there was no statistical difference in the rates of SMA detection with the use of hand motor seeding for rs-fMRI. Within the larger cohort of patients with brain tumor, the detection rate of SMA activation was significantly higher when selecting the hand motor region as the seeding area instead of the orofacial motor region. This result indicates stronger correlation between hand motor areas and the SMA compared with the orofacial motor areas. This difference may be due to the variable connectivity of the dorsalversus-ventral motor systems; indeed, when independent component analysis is performed, these 2 systems routinely are separated into different components with current acquisition and analysis methods. Underlying differences in dynamic connectivity may drive this phenomenon, with the ventral motor system demonstrating higher homotopic connectivity compared with the dorsal motor system. ${ }^{28}$ In addition, our study demonstrated higher rates of SMA detection when using seeds from the bilateral hand motor areas compared with the unilateral hand motor areas, conceivably also due to potential differences in dynamic connectivity between the homotopic regions, which, when averaged, result in improved detection of the SMA. While the SMA is known to lateralize with lateralizing tasks, signal from the unilateral ROIs may be less robust to demonstrate the SMA with the standard methods used in our study. Further studies may investigate whether incorporating dynamic connectivity can improve SMA detection, as well as determine whether the SMA may be lateralized 


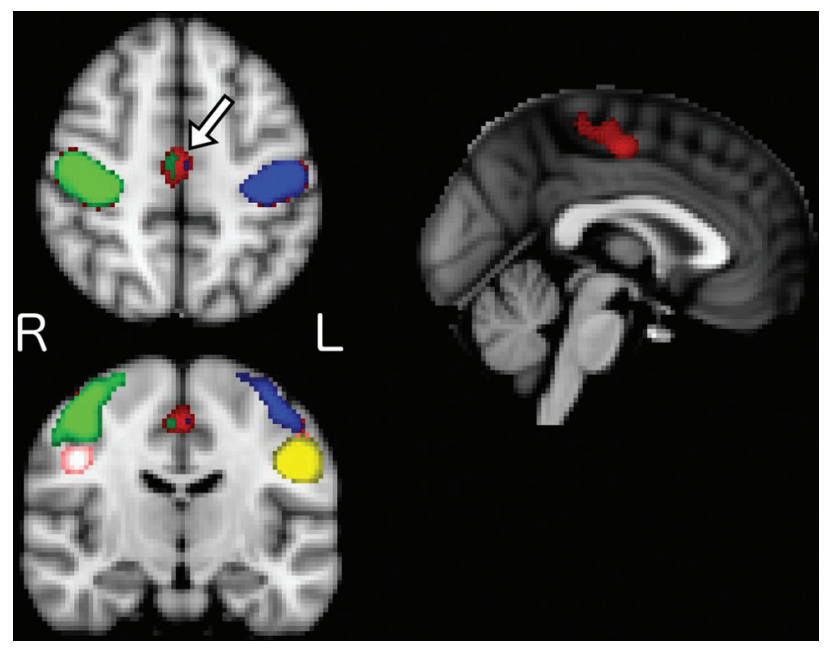

FIGURE. Mean group correlation maps across different seed locations for patients with brain tumor (bilateral hand motor seed in red, left hand motor in blue, right hand motor in green, left tongue motor in yellow, and right tongue motor in pink; please note that left and right denote the cerebral hemisphere of the seeds and thus represent contralateral functional regions). The greatest correlation in the supplementary motor area (arrow on the axial image) is seen with bilateral hand motor seeds. To a much lesser extent in this group visualization, left hand and right hand motor seeds demonstrate correlation in the SMA; however, neither tongue motor seed does so. $\mathrm{R}$ indicates right; $\mathrm{L}$, left.

Table 2: Number of cases with identifiable SMA by seeding areas

\begin{tabular}{lccccc}
\hline & \multicolumn{2}{c}{ Patient } & & \multicolumn{2}{c}{ Control } \\
\cline { 2 - 3 } \cline { 6 - 6 } \multicolumn{1}{c}{ ROI } & +SMA & -SMA & & +SMA & -SMA \\
Hand (right) & $50(75.8)$ & $16(24.2)$ & & $38(90.5)$ & $4(9.5)$ \\
Hand (left) & $50(75.8)$ & $16(24.2)$ & & $34(81.0)$ & $8(19.0)$ \\
Hand (both) & $63(95.5)$ & $3(4.5)$ & & $40(95.2)$ & $2(4.8)$ \\
Orofacial (right) & $17(25.8)$ & $49(74.2)$ & & $14(33.3)$ & $28(66.7)$ \\
Orofacial (left) & $18(27.3)$ & $48(72.7)$ & & $9(21.4)$ & $33(78.6)$ \\
Orofacial (both) & $23(34.8)$ & $43(65.2)$ & & $19(45.2)$ & $23(54.8)$ \\
\hline
\end{tabular}

Note:- +SMA indicates that the SMA was identified; -SMA, the SMA was not identified.

Table 3: Identifiable SMA percentages compared across ROIs in patients with brain tumor

\begin{tabular}{|c|c|c|c|}
\hline Target Group & $\begin{array}{l}\text { Percentage } \\
\text { Identified } \\
(95 \% \mathrm{CI})\end{array}$ & $\begin{array}{l}\text { Reference Group } \\
\text { Percentage } \\
\text { Identified } \\
(95 \% \mathrm{Cl})\end{array}$ & $\begin{array}{c}P \\
\text { Value }\end{array}$ \\
\hline Hand & & Hand (both) & \\
\hline Hand (right) & $75.8(63.4-85.1)$ & 95.5 (86.4-98.8) & $<.001^{\mathrm{b}}$ \\
\hline Hand (left) & $75.8(63.4-85.1)$ & $95.5(86.4-98.8)$ & $<.001^{\mathrm{b}}$ \\
\hline Orofacial & & Orofacial (both) & \\
\hline Orofacial (right) & $25.8(16.1-38.2)$ & $34.8(23.8-47.7)$ & .074 \\
\hline Orofacial (left) & $27.3(17.4-39.8)$ & $34.8(23.8-47.7)$ & .041 \\
\hline Hand (both) & $95.5(86.4-98.8)$ & $34.8(23.8-47.7)$ & $<.001^{\mathrm{b}}$ \\
\hline
\end{tabular}

using unilateral ROIs with improved scanning, processing, and analysis methods.

Regarding various rs-fMRI techniques, prior studies have demonstrated high correspondence in overall connectivity results between seed-based analysis and independent component analysis methods. ${ }^{29}$ Seed-based analysis requires a priori selection of ROIs, which can be somewhat challenging and may slightly differ
Table 4: Identifiable SMA percentages compared across ROIs in the control group

\begin{tabular}{|c|c|c|c|}
\hline Target Group & $\begin{array}{l}\text { Percentage } \\
\text { Identified } \\
(95 \% \mathrm{Cl})\end{array}$ & $\begin{array}{l}\text { Reference Group } \\
\text { Percentage } \\
\text { Identified } \\
(95 \% \mathrm{Cl})\end{array}$ & $\begin{array}{c}P \\
\text { Value }^{a}\end{array}$ \\
\hline Hand & & Hand (both) & \\
\hline Hand (right) & $90.5(76.4-96.9)$ & $95.2(82.6-99.2)$ & .480 \\
\hline Hand (left) & $81.0(65.4-90.8)$ & $95.2(82.6-99.2)$ & .041 \\
\hline Orofacial & & Orofacial (both) & \\
\hline Orofacial (right) & $33.3(20.0-49.6)$ & $45.2(30.2-61.2)$ & .074 \\
\hline Orofacial (left) & $21.4(10.8-37.2)$ & $45.2(30.2-61.2)$ & $.004^{\mathrm{b}}$ \\
\hline Hand (both) & $95.2(82.6-99.2)$ & $45.2(30.2-61.2)$ & $<.001^{\mathrm{b}}$ \\
\hline
\end{tabular}

${ }^{\text {a }}$ From the McNemar $\chi^{2}$ test.

b Significant.

Table 5: Number of cases with identifiable SMA across ROls, control vs brain tumor group

\begin{tabular}{lccc}
\hline & \multicolumn{2}{c}{$\begin{array}{c}\text { SMA Identified } \\
\text { (No.) (\%) }\end{array}$} & P \\
\cline { 2 - 3 } & Control & Brain Tumor & Value $^{\text {a }}$ \\
\hline Total & $42(100.0)$ & $66(100.0)$ & \\
Hand (right) & $38(90.5)$ & $50(75.8)$ & .055 \\
Hand (left) & $34(81.0)$ & $50(75.8)$ & .527 \\
Hand (both) & $40(95.2)$ & $63(95.5)$ & 1.000 \\
Orofacial (right) & $14(33.3)$ & $17(25.8)$ & .513 \\
Orofacial (left) & $9(21.4)$ & $18(27.3)$ & .649 \\
Orofacial (both) & $19(45.2)$ & $23(34.8)$ & .315 \\
\hline
\end{tabular}

${ }^{a}$ Fisher exact test.

between study protocols. In particular, brain shifts or gyral expansion in patients with large brain tumors may cause a large amount of distortion that precludes accurate placement of seed ROIs. Independent component analysis overcomes this specific problem; however, identification of specific network subcomponents may be limited due to the number of targeted independent component analysis components. This may be the limiting factor in another study in which the SMA was inconsistently found using independent component analysis, which was thought to be due to network fragmentation related to independent component analysis order. ${ }^{11}$ Our approach demonstrates that despite possible mass effect, the SMA can be readily identified in nearly all patients using well-known anatomic landmarks that are not in the immediate vicinity of the area of interest. The question of SMA localization for operative planning is relevant when tumors are within or adjacent to the expected location of the SMA. Therefore, in most cases, the primary motor cortex may still be effectively used for ROI placement as shown in this study.

There are several limitations to our study, one being that findings were not compared directly with the criterion standard of direct cortical stimulation. However, SMA evaluation using DCS is more challenging compared with evaluation of the primary motor regions; indeed, at our institution, neurosurgical requests for primary motor function localization using fMRI are far less common compared with requests for SMA localization, due to the difficulty of obtaining accurate results for the latter using DCS. For identification of the SMA, no independent assessment was performed between the 2 neuroradiologists, and consensus evaluation was used. In addition, no quantitative analysis was performed; while there have been attempts to standardize thresholding of fMRI (eg, using a percentage of the maximum local 
signal) ${ }^{30}$ there are no current established standards. Our qualitative method, however, actually represents a more real-world scenario, in which localization and thresholding of the SMA in tbfMRI are performed using clinical judgment. Finally, the images of the patients with brain tumor were acquired on different scanners than for the healthy controls, potentially introducing scanner variability as a confounder in our results.

\section{CONCLUSIONS}

We demonstrate that rs-fMRI can be reliably used to identify the SMA in most patients with brain tumors, to the same degree as subjects with no brain lesions. We also demonstrate that the use of bilateral hand motor regions for seed placement is superior in identifying the SMA compared with unilateral hand motor or orofacial motor region seeding.

Disclosures: Jay J. Pillai—UNRELATED: Royalties: Springer Science \& Business Media, Elsevier, Comments: royalty for books.

\section{REFERENCES}

1. Ojemann G, Ojemann J, Lettich E, et al. Cortical language localization in left, dominant hemisphere: an electrical stimulation mapping investigation in 117 patients. 1989. J Neurosurg 2008;108: 411-21 CrossRef Medline

2. Matthews PM, Honey GD, Bullmore ET. Applications of fMRI in translational medicine and clinical practice. Nat Rev Neurosci 2006; 7:732-44 CrossRef Medline

3. Sair HI, Yahyavi-Firouz-Abadi N, Calhoun VD, et al. Presurgical brain mapping of the language network in patients with brain tumors using resting-state fMRI: Comparison with task fMRI. Hum Brain Mapp 2016;37:913-23 CrossRef Medline

4. Schneider FC, Pailler M, Faillenot I, et al. Presurgical assessment of the sensorimotor cortex using resting-state fMRI. AJNR Am J Neuroradiol 2016;37:101-07 CrossRef Medline

5. Branco P, Seixas D, Deprez S, et al. Resting-state functional magnetic resonance imaging for language preoperative planning. Front Hum Neurosci 2016;10:11 CrossRef Medline

6. Bizzi A, Blasi V, Falini A, et al. Presurgical functional MR imaging of language and motor functions: validation with intraoperative electrocortical mapping. Radiology 2008;248:579-89 CrossRef Medline

7. Biswal B, Yetkin FZ, Haughton VM, et al. Functional connectivity in the motor cortex of resting human brain using echo-planar MRI. Magn Reson Med 1995;34:537-41 CrossRef Medline

8. Palanca BJ, Mitra A, Larson-Prior L, et al. Resting-state functional magnetic resonance imaging correlates of sevoflurane-induced unconsciousness. Anesthesiology 2015;123:346-56 CrossRef Medline

9. Sämann PG, Wehrle R, Hoehn D, et al. Development of the brain's default mode network from wakefulness to slow wave sleep. Cereb Cortex 2011;21:2082-93 CrossRef Medline

10. Dierker D, Roland JL, Kamran M, et al. Resting-state functional magnetic resonance imaging in presurgical functional mapping: sensorimotor localization. Neuroimaging Clin NAm 2017;27:621-33 CrossRef Medline

11. Kokkonen SM, Nikkinen J, Remes J, et al. Preoperative localization of the sensorimotor area using independent component analysis of resting-state fMRI. Magn Reson Imaging 2009;27:733-40 CrossRef Medline

12. Liu H, Buckner RL, Talukdar T, et al. Task-free presurgical mapping using functional magnetic resonance imaging intrinsic activity. J Neurosurg 2009;111:746-54 CrossRef Medline
13. Fontaine D, Capelle L, Duffau H. Somatotopy of the supplementary motor area: evidence from correlation of the extent of surgical resection with the clinical patterns of deficit. Neurosurgery 2002;50: 297-303, discussion 303-05 Medline

14. Russell SM, Kelly PJ. Incidence and clinical evolution of postoperative deficits after volumetric stereotactic resection of glial neoplasms involving the supplementary motor area. Neurosurgery 2003;52:506-16; discussion 515-16 CrossRef Medline

15. Vassal M, Charroud C, Deverdun J, et al. Recovery of functional connectivity of the sensorimotor network after surgery for diffuse low-grade gliomas involving the supplementary motor area. J Neurosurg 2017;126:1181-90 CrossRef Medline

16. Mitchell TJ, Hacker CD, Breshears JD, et al. A novel data-driven approach to preoperative mapping of functional cortex using resting-state functional magnetic resonance imaging. Neurosurgery 2013;73:969-82, discussion 982-83 CrossRef Medline

17. Cochereau J, Deverdun J, Herbet G, et al. Comparison between resting state fMRI networks and responsive cortical stimulations in glioma patients. Hum Brain Mapp 2016;37:3721-32 CrossRef Medline

18. Landman BA, Huang AJ, Gifford A, et al. Multi-parametric neuroimaging reproducibility: a 3-T resource study. Neuroimage 2011;54: 2854-66 CrossRef Medline

19. Oldfield RC. The assessment and analysis of handedness: the Edinburgh inventory. Neuropsychologia 1971;9:97-113 CrossRef Medline

20. McAuliffe MJ, Lalonde FM, McGarry D, et al. Medical image processing, analysis and visualization in clinical research. In: Proceedings of the IEEE Symposium on Computer-Based Medical Systems, Bethesda, Maryland. July 26-27, 2001:381-86

21. Mazaika PK, Fumiko H, Glover GH, et al. Methods and software for fMRI analysis for clinical subjects. In: Proceedings of the Annual Meeting of the Organization for Human Brain Mapping, San Francisco, California; June 15, 2009

22. Behzadi Y, Restom K, Liau J, et al. A component based noise correction method (CompCor) for BOLD and perfusion based fMRI. Neuroimage 2007;37:90-101 CrossRef Medline

23. Chauvel PY, Rey M, Buser P, et al. What stimulation of the supplementary motor area in humans tells about its functional organization. Adv Neurol 1996;70:199-209 Medline

24. Fried I. Electrical stimulation of the supplementary sensorimotor area. Adv Neurol 1996;70:177-85 Medline

25. Ibe $\mathrm{Y}$, Tosaka M, Horiguchi K, et al. Resection extent of the supplementary motor area and post-operative neurological deficits in glioma surgery. Br J Neurosurg 2016;30:323-29 CrossRef Medline

26. Qiu TM, Yan CG, Tang WJ, et al. Localizing hand motor area using resting-state fMRI: validated with direct cortical stimulation. Acta Neurochir (Wien) 2014;156:2295-302 CrossRef Medline

27. Hou BL, Bhatia S, Carpenter JS. Quantitative comparisons on hand motor functional areas determined by resting state and task BOLD fMRI and anatomical MRI for pre-surgical planning of patients with brain tumors. Neuroimage Clin 2016;11:378-87 CrossRef Medline

28. Syed MF, Lindquist M, Pillai JJ, et al. Dynamic functional connectivity states between the dorsal and ventral sensorimotor networks revealed by dynamic conditional correlation analysis of resting state functional magnetic resonance imaging. Brain Connect 2017;7: 635-42 CrossRef Medline

29. Rosazza C, Minati L, Ghielmetti F, et al. Functional connectivity during resting-state functional MR imaging: study of the correspondence between independent component analysis and regionof-interest-based methods. AJNR Am J Neuroradiol 2012;33:180-87 CrossRef Medline

30. Voyvodic JT. Reproducibility of single-subject fMRI language mapping with AMPLE normalization. J Magn Reson Imaging 2012;36: 569-80 CrossRef Medline 